ON SUM COMPOSITTION OF FRACTIONAL

FACTORIAL DESIGNS

by

J. Joiner, B. L. Raktoe and W. T. Federer

Cornell University and University of Guelph

Statistical Series 1973-11

University of Guelph 


\section{ON SUM COMPOSITION OF FRACTIONAL}

\section{FACTORIAL DESIGNS}

by

J. Joiner, B. L. Raktoe and W. T. Federer

Cornell University and University of Guelph

\section{ABSTRACT}

Combinatorial extension and composition methods have been extensively used in the construction of block designs. One of the composition methods, namely the direct product or Kronecker product method was utilized by chakravarti [1956] to produce certain types of fractional factorial designs. The present paper shows how the direct sum operation can be utilized in obtaining from initial fractional factorial designs for two seperate symmetrical factorials a fractional factorial design for the corresponding asymmetrical factorial. Specifically, we provide some results which are useful in the construction of non-singular fractional factorial designs via the direct sum composition method. In addition a modified direct sum method is discussed and the consequences of imposing orthogonality are explored. 


\section{ON SUM COMPOSITION OF FRACTIONAL}

FACTORIAL DESIGNS ${ }^{1]}$

by

J. Joiner, B. L. Raktoe and W. T. Federer

Cornell University and University of Guelph

\section{INTRODUCTION}

In design theory there are well known algebraic operations which lead to new designs, when we start out from a set of initial designs. For example, the direct product (or Kronecker product) operation was used by Chakravarti [1956] to produce fractional factorial designs for the asymmetrical factorial. The designs developed by him through this method did not relate to arbitrary initial factorial designs. These initicl deisgns specifically arose from the existence of orthogonal arrays, which were much earlier shown to be qujte useful in factorial design theory by Rao [1947].

To illustrate the direct product method we reproduce the following example, which follows immediately from theorem I of chakravarti's [1956] paper. The orthogonal arrays $D_{1}^{*}$ and ${ }^{*} ?$ below are orthogonal main effect plans in $\mathrm{N}_{1}=4$ and

1 licarireh :supported by NRC crant No. A7200 and by NIH Grant No. $!-K 01-(: M-! ; 900$.

Keywordsi and phrases. Tractional replication, sum composition, nonsinguldrity, similar designs, orthogonal fractions. 
$\mathrm{N}_{2}=9$ treatment combinations for the $2^{3}$ and $3^{4}$ factorials respectively.

$\mathrm{D}_{1}^{*}$

000

$(1 . I)$

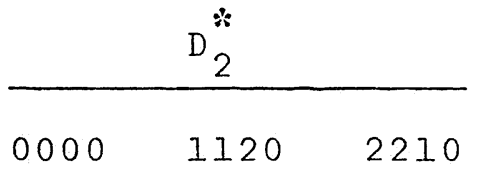

$0112 \quad .1202$

$0221 \quad 2022$

$1011 \cdot 2101$

011

The direct product design $\mathrm{D}_{1}^{*} \otimes \mathrm{D}_{2}^{*}$ in $\mathrm{N}_{1} \mathrm{~N}_{2}=36$ treatment combinations provides orthogonal estimates of not only the main effects but also of the two factor interaction of one 2-level factor with one 3 -level factor for the $2^{3} \times 3^{4}$ factorial under the assumption that all other effects are negligible.

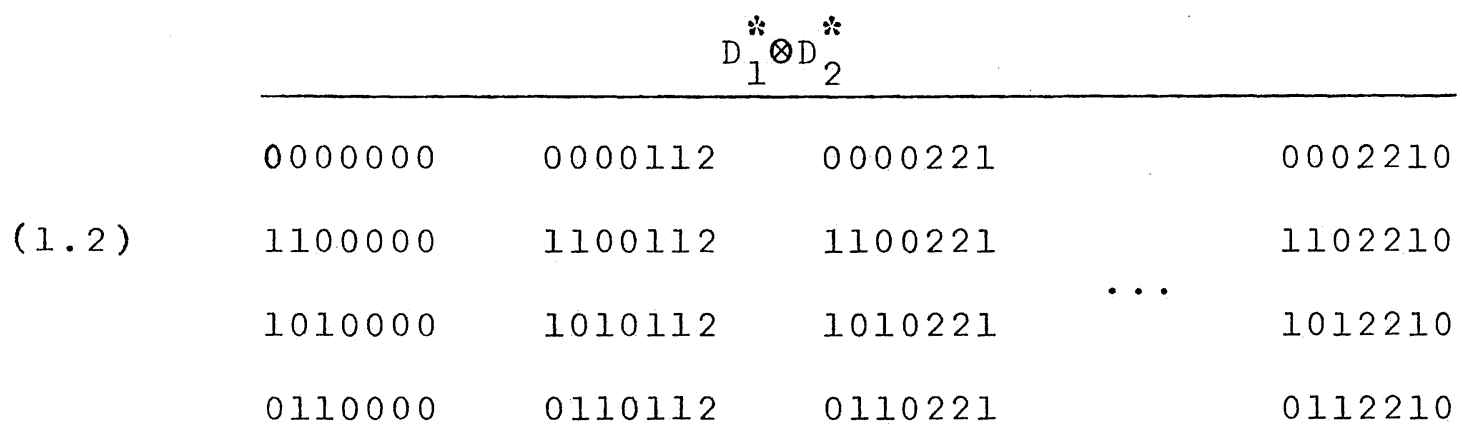

In addition to the above type of designs there is a need to spell out the details of the direct product method for arbitrary initial desings and given arbitrary parameters under virious; astiumptions on the total parametric vector. Such initial do:;igns would encompass resolution III, IV and V de: i gnes. 
In some settings (especially when the orthogonality condition is dropped) the resultant direct product design might be uneconomical from the viewpoint of number of treatment combinations. Thus, in the previous example, if main effects are the only ones of interest under the assumption that all interactions are negligible, it is clear that 36 treatment combinations are too many for estimation purposes. This is so because for the $2^{3} \times 3^{4}$ factorial we need only 12 treatment combinations to form a main effect plan if no estimate of the error variance is desired. If it is desirable to have an estimate of the error variance as well then one or more treatment combinations can be added to the plan or one or more treatment combinations can be repeated in the plan.

To obtain economical fractions we can resort to a different operation altogether, e.g. we can compose two initial designs using the direct sum operation. Before taking a formal approach consider the main effect designs $D_{1}$ and $D_{2}$ consisting of $\mathrm{N}_{1}=4$ and $\mathrm{N}_{2}=6$ treatment combinations for the $k_{1}^{\mathrm{m}}{ }^{1}=2^{2}$ and $\mathrm{k}_{2}{ }^{2}=3^{2}$ factorials respectively:

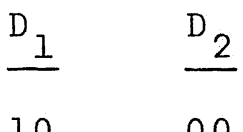

$(1.3)$

01,22

$11 \quad 10$

$11 \quad 12$ 
It is easily verified, that the design $D_{1} \oplus D_{2}$ below is a nonsingular main effect plan in $\mathrm{N}_{1}+\mathrm{N}_{2}=4+6=10$ treatment combina-

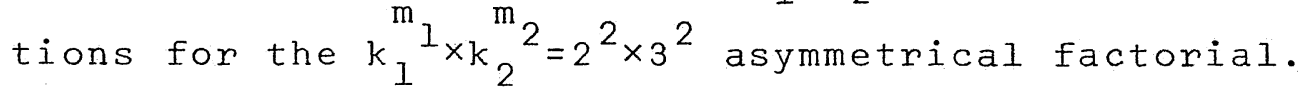

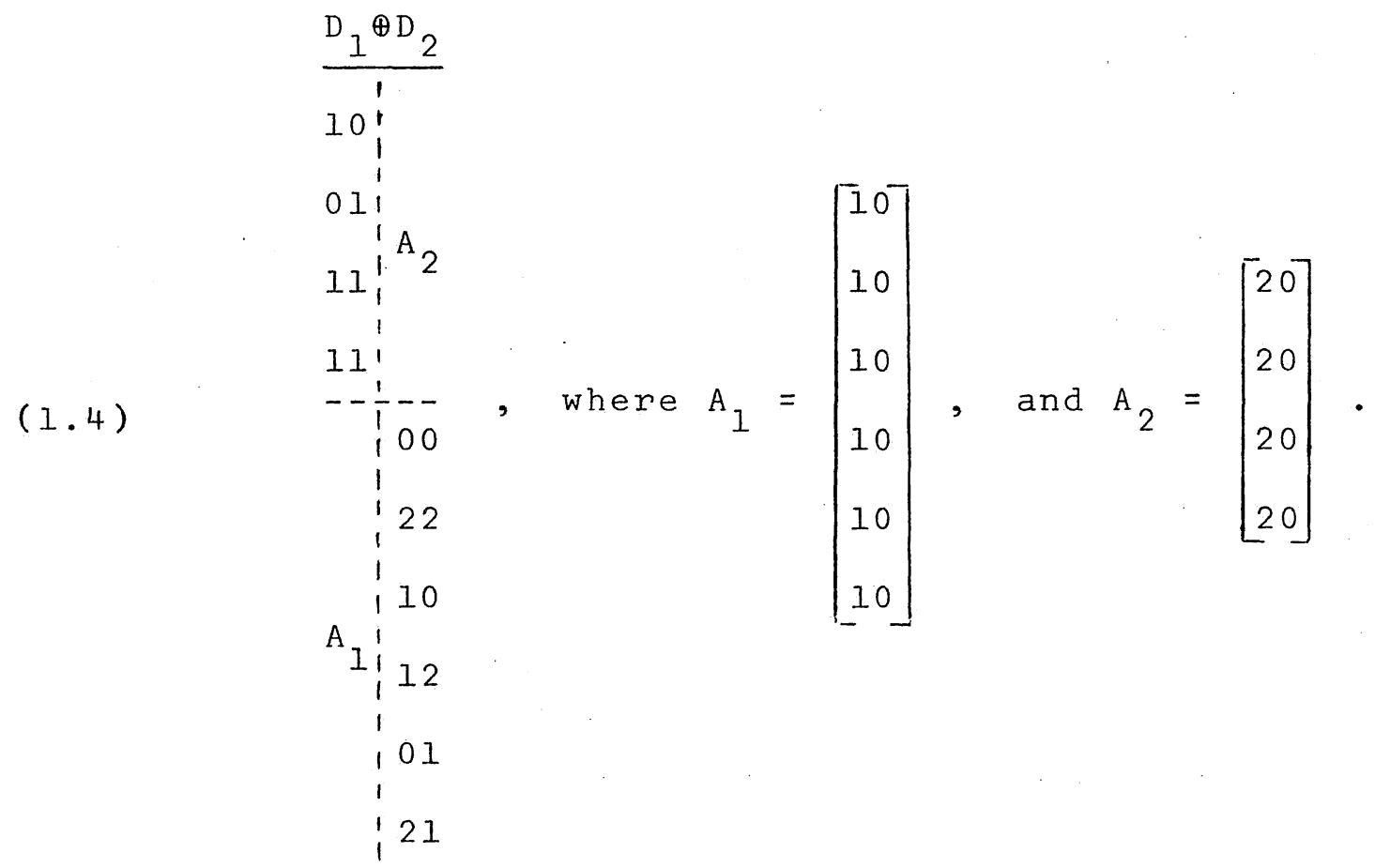

The operation involved in producing this design is clearly a direct sum type of operation, which we will call compactly direct sum composition. It is clear that the crucial part in using this method is the specification of the matrices $A_{I}$ and $\Lambda_{2}$. 'l'he choice of these will depend on what kind of properties one wishes to impose on the resulting design, given cert.ain propertios on the initial designs.

In the following sections we explore this new method in more detail and show how in some settings it always produces a clesign for the asymmetrical $k_{1}^{m^{1} \times k_{2}{ }^{m}}$ factorial given the 
initial designs for the $k_{1}^{\mathrm{m}}$ and $k_{2}^{\mathrm{m}}{ }^{2}$ factorials.

\section{PRELIMINARY DEFINITIONS AND NOTATIONS}

To make this paper relatively self-contained we introduce some of the basic notions of fractional replication. Consider the symmetric $k_{1} \times k_{2} \times \ldots \times k_{t}$ factorial consisting of $t$ factors the $i-t h$ one having $k_{i}$ levels. Let $T$ denote the set of $N=\prod_{i=1}^{t} k_{i}$ treatment combinations.

DEFINITION 2.1. A factorial arrangement $\Gamma$ with parameters $k_{1}, k_{2}, \ldots, k_{t} ; m ; n ; r_{1}, r_{2}, \ldots, r_{N}$ is defined to be a collection of $n=\sum_{j=1} r_{j}$ treatments of $T$ such that the j-th treatment in $\mathrm{T}$ has multiplicity (= replication number) $r_{j}$, and $m$ is the number of nonzero $r_{j}$ 's.

DEFINITION 2.2 A factorial arrangement is said to be complete if $r_{j}>0$ for all $j$.

DEFINITION 2.4. A factorial arrangement is said to be a fractional factorial arrangement, or simply a fractional replicate, if some but not all $r_{j}>0$.

l.et $\theta$ denote the $N \times 1$ vector of single degree of freedom paramoters; (also referred to as factorial effects) and let $y_{h}$ le an observation at treatment $h$. The model we associate 
with $T$ and $\theta$ is:

$$
E\left[Y_{T}\right]=X_{T} \theta, \quad \operatorname{Cov}\left[Y_{T}\right]=\sigma^{2} I
$$

where an element of the $N \times I$ vector of observations $\mathrm{Y}_{\mathrm{T}}$ is $\mathrm{y}_{\mathrm{h}}$ such that:

$$
\text { (2.2) } E\left[y_{h}\right]=\theta^{\prime} f(h) \text {. }
$$

Here $f$ is an $N$-vector of $k$ real valued known functions on the h's in $T$. We assume that the design matrix $X_{T}$ is in orthonormal form, i.e. $X_{T}^{\prime} X_{T}=I_{N}$. A familiar model of type (2.1) is the orthogonal-polynomial model.

$$
\text { Corresponding to a factorial arrangement } \Gamma \text { the model }
$$
for the $n \times I$ observation vector $Y_{\Gamma}$ as induced by (2.1) is equal to:

$$
E\left[Y_{\Gamma}\right]=X_{\Gamma} \theta
$$

where $x_{\Gamma}$ i.s an $n \times N$ matrix simply read off from $x_{T}$ taking repetitions into account.

To make the notation and forthcoming discussion simpler,

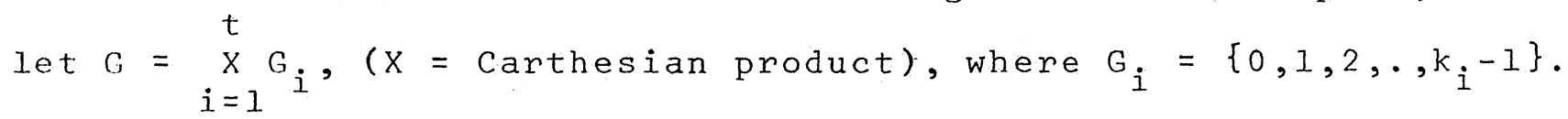


Identify the elements of $G_{i}$ with the levels of the $i-t h$ factor, so that $G$ is a representation of $T$. With this representation a treatment or treatment combination $g$ is then an element of G. Hence equations (2.1) and (2.2) may be rewritten as $E\left[Y_{G}\right]=X_{G} \theta$ and $E\left[y_{g}\right]=\theta^{\prime} f(g)$, where $X_{G}=X_{T}$ and $f(g)=f(h)$. Thus when dealing with a fractional replicate $\Gamma$ one is dealing with a collection of g's from G. Further,

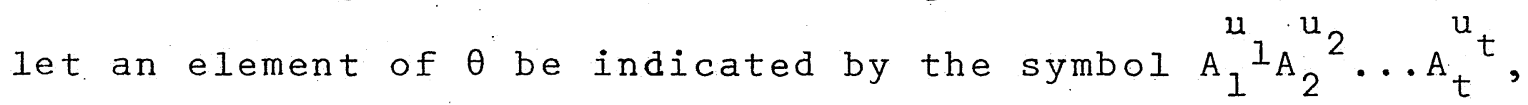
where $\left(u_{1}, u_{2}, \ldots, u_{t}\right)$ is an element of $G$. This means that we have brought both the set of treatment combinations $\mathrm{T}$ and the set of factorial effects $\theta$ into one to one correspondence with $G$.

From the experimenter's viewpoint the most realistic partioning of the vector $\theta$ is the following:

$$
\theta^{\prime}=\left(\theta_{1}^{\prime}: \theta_{2}^{\prime}: \theta_{3}^{\prime}\right)
$$

where $\theta_{1}$ is an $N_{1}$-vector to be estimated, $\theta_{2}$ is an $N_{2}$-vector not of interest for estimation and not assumed to be known, and $\theta_{3}$ is an $\mathrm{N}_{3}$-vector assumed to be negligible (i.e. equal to zero), $1 \leq \mathrm{N}_{1} \leq \mathrm{N}, \quad 0 \leq \mathrm{N}_{2} \leq \mathrm{N}-1,0 \leq \mathrm{N}_{3} \leq \mathrm{N}-1$ with $\mathrm{N}_{1}+\mathrm{N}_{2}+\mathrm{N}_{3}=\mathrm{N}$. The above partioning then leads to the following four cases: 
$(2.5)$

$$
\text { (i) } \quad \mathrm{N}_{1}=\mathrm{N}, \mathrm{N}_{2}=\mathrm{N}_{3}=0
$$$$
\text { (ii) } \quad \mathrm{N}_{2}=0, \mathrm{~N}_{3} \neq 0
$$

$$
\text { (iii) } \mathrm{N}_{2} \neq 0, \mathrm{~N}_{3} \neq 0
$$$$
\text { (iv) } \mathrm{N}_{2} \neq 0, \mathrm{~N}_{3}=0
$$

It is well known that case (ii) gives rise to odd resolution problems and case (iii) to even resolution problems. Case (i) leads to BLU estimation problem of $\theta$ and to response surface fitting problems while cases (iii) and (iv) bring about the biased estimation problems.

\section{THE SUM COMPOSITION METHOD}

Consider two separate symmetric factorials, i.e. the $\mathrm{k}_{1} \times \mathrm{k}_{1} \times \ldots \times \mathrm{k}_{1}=\mathrm{k}_{1}^{\mathrm{m}_{1}}$ and the $\mathrm{k}_{2} \times \mathrm{k}_{2} \times \ldots \times \mathrm{k}_{2}=\mathrm{k}_{2}^{\mathrm{m}_{2}}$ factorials and consider for each factorial the partitioning in (2.4). Explicitly let the ${ }^{{ }^{m}}{ }_{2}^{i} \times l$ parametric vector $\beta_{i}$ be partitioned as:

$$
\beta_{i}^{\prime}=\left(\beta_{i 1}^{\prime} \vdots \beta_{i 2}^{\prime} \vdots \beta_{i 3}^{\prime}\right), \quad i=1,2
$$

where $\beta_{i l}$ is the $p_{i l} \times l$ vector of parameters to be estimated, $\beta_{i 2}$ is the $p_{i 2} \times 1$ vector of parameters not of interest and not assumed to be zero, and, $\beta_{i 3}$ is the $\left(k_{i}^{m_{i}}-p_{i I}-p_{i 2}\right) \times 1$ vector of parameters assumed to be zero. We assume the first element of both $\beta_{11}$ and $\beta_{2 l}$ in respectively the $k_{l}{ }_{1}$ and $k_{2}{ }^{m}$ 
factorials to be equal to the mean $\mu$. Also we limit ourselves in this section to the most popular case, i.e. the odd resolution case, where $\mathrm{p}_{12}=\mathrm{p}_{22}=0$. Note that this is case (ii) of the previous section for two separate symmetrical factorials.

Let $D_{i}=1,2$, be a design consisting of $N_{i}$ treatment combinations from the $k_{i}^{{ }_{i}}$ factorial such that the vector $\beta_{i}$ is estimable. Further let $\beta_{11} \cup \beta_{21}$ be the $\left(p_{11}+p_{21}-1\right) \times 1$ parametric vector whose entries are elements of the union of $\beta_{11}$ and $\beta_{21}$, when these are considered as sets of parameters

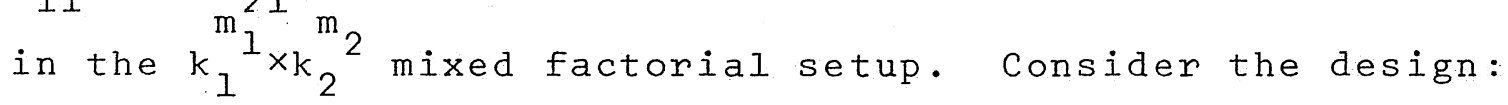

$(3.1)$

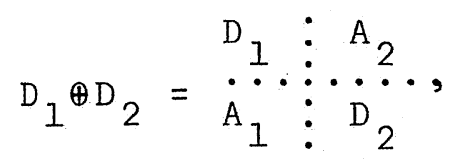

where $A_{1}$ is $N_{2} \times_{1}$ and $A_{2}$ is $N_{1} \times m_{2}$, and the rows of $A_{i}$ are treatment combinations from the $\mathrm{k}_{i}{ }^{i}$ factorial. We desire a choice of $A_{1}$ and $A_{2}$ such that the resulting design provides unbiased estimates for the elements of $\beta_{11} \cup \beta_{21}$.

DLITITION 3.1. Given designs $D_{1}$ and $D_{2}$ such that $\beta_{11}$ and $\beta_{2 l}$ are estimable in the symmetrical factorials $k_{l}{ }_{l}$ and $k_{2}{ }^{\prime \prime}$ respectively, then a design $\mathrm{D}_{1} \oplus \mathrm{D}_{2}$ consisting of $\mathrm{N}_{1}+\mathrm{N}_{2}$ treatment combinations such that $\beta_{11} \cup \beta_{21}$ is estimable in the ${ }_{k}{ }_{1}{ }^{1} \times k_{2}^{m}{ }^{2}$ mixed factorial, is said to be obtained using the direct sum composition of design $D_{1}$ and $D_{2}$. 
It is clear that the crucial part in coming up with a design $D_{1} \oplus D_{2}$ such that $\beta_{11} \cup \beta_{21}$ is estimable is the specification of the matrices $A_{1}$ and $A_{2}$, because they will have to guarantee the nonsingularity of the underlying design matrix of $\mathrm{D}_{1} \oplus \mathrm{D}_{2}$. In other words, the selection of $A_{1}$ and $A_{2}$ should be such that rank of the design matrix of $D_{1} \oplus D_{2}$ is equal to $\mathrm{p}_{11}+\mathrm{p}_{21}{ }^{-1}$. Before providing some nonsingular constructions of direct sum designs, we first proceed to prove an algebraic theorem which is of a fundamental nature.

Let $D_{1} \oplus D_{2}$ be a direct sum design, then its design ma-

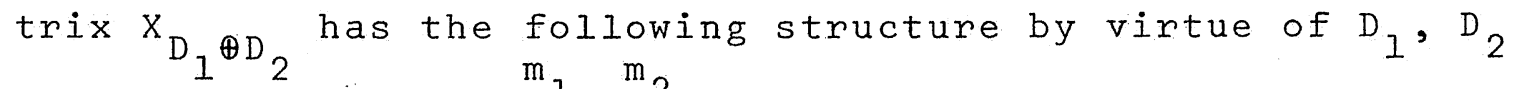

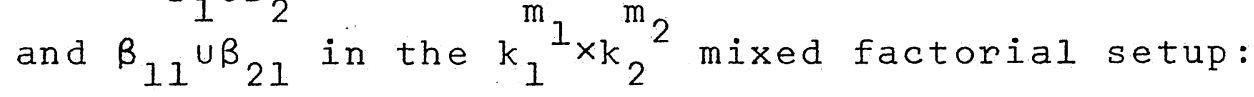

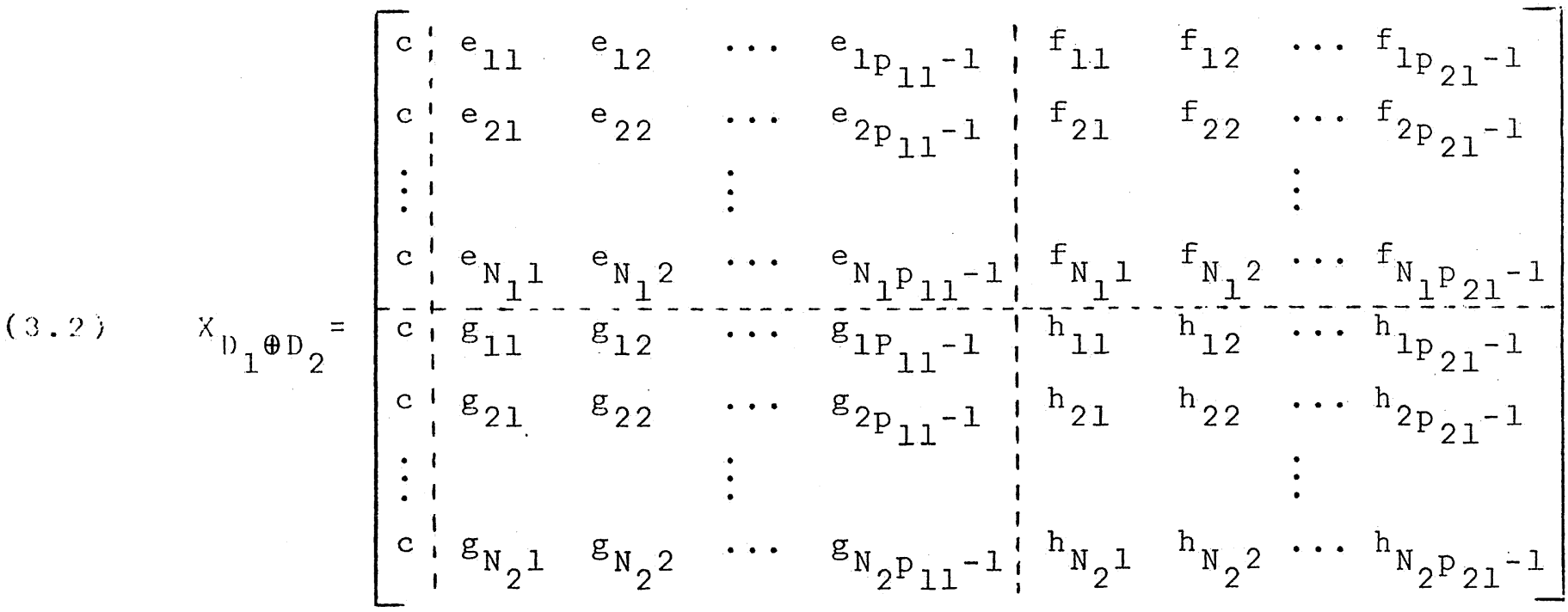


( 3.3 )

$$
=\left[\begin{array}{c:c:c}
{ }^{C l}{ }_{N} & E & F \\
\hdashline c^{1}{ }_{N} & G & H
\end{array}\right] .
$$

Note that tre matrices $E$ and $H$ are essentially determined by $D_{1}$ and $D_{2}$ and $B_{11} \cup \beta_{21}$ in the context of the $k_{1}^{{ }^{m}{ }^{l} \times k_{2}{ }^{m}} \operatorname{mixed}$ factorial. Let us now form the following vectors of order $\mathrm{N}_{1}+\mathrm{N}_{2}:$

$(3.4)$

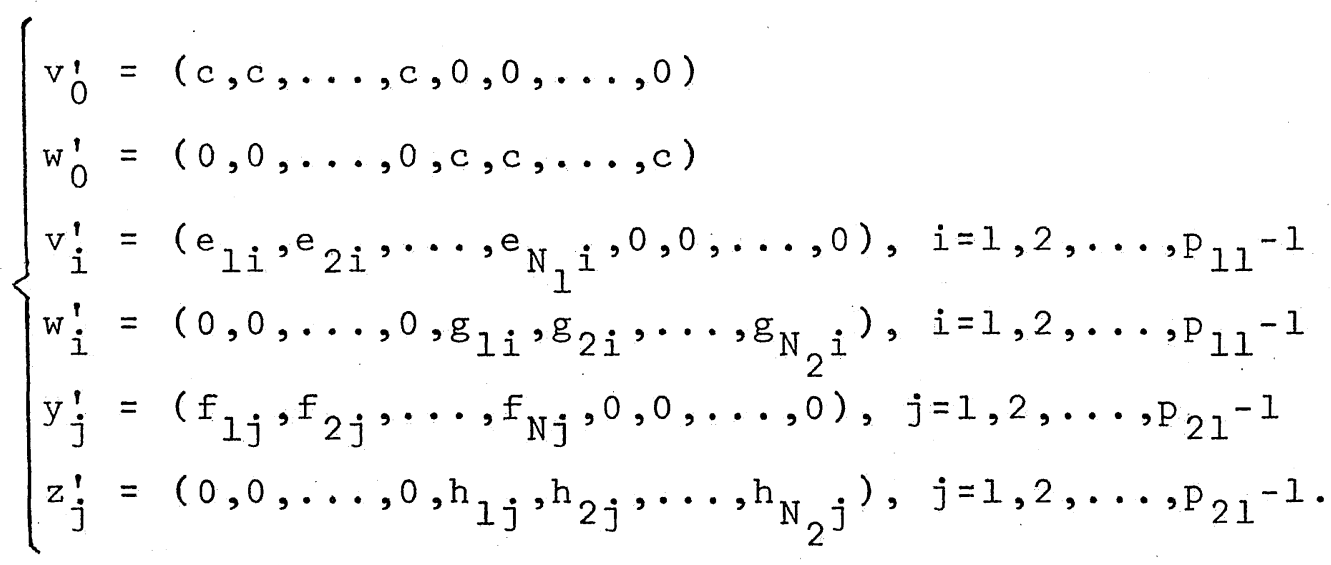

Denote the columns of $x_{D_{1} \oplus D_{2}}$ by $x_{0}, x_{1}, x_{2}, \ldots, x_{p_{11}}, x_{1}^{*}, x_{2}^{*}, \ldots$, $\mathrm{x}_{\mathrm{p}_{21}}^{*}$, then it is seen that:

$(3.5)$

$$
\left\{\begin{array}{l}
x_{0}=v_{0}^{\prime}+w_{0}^{\prime} \\
x_{i}^{\prime}=v_{i}^{\prime}+w_{i}^{\prime}, i=1,2, \ldots, p_{11}-1 \\
x_{j}^{*^{\prime}}=y_{j}^{\prime}+z_{j}^{\prime}, j=1,2, \ldots, p_{21}-1
\end{array}\right.
$$


Let $X$ be the subspace spanned by the vectors $x_{0}, x_{1}, x_{2}, \ldots$, $\mathrm{x}_{\mathrm{p}_{11}-1}$ and $X^{*}$ the subspace spanned by $\mathrm{x}_{1}^{*}, \mathrm{x}_{2}^{*}, \ldots, \mathrm{x}_{\mathrm{p}_{21}}^{* 1}$, then the dimension of the space $x+x^{*}$ spanned by the two sets of vectors is equal to:

$$
\operatorname{dim}\left(x+x^{*}\right)=\operatorname{dim} x+\operatorname{dim} x^{*}-\operatorname{dim} x \cap x^{*}
$$

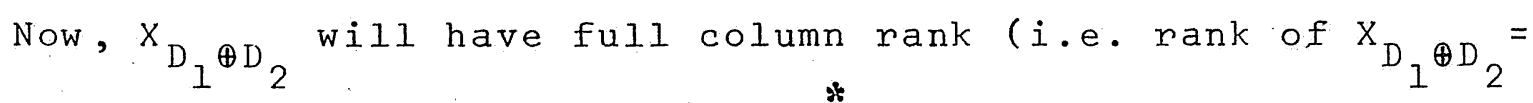
$\left.\mathrm{p}_{11}+\mathrm{p}_{21}{ }^{-1}\right)^{2}$ if and only if dim $\chi \cap x^{*}$ is equal to zero. On the other hand for a vector $x$ to be in $x \cap x^{*}$, it must be a linear combination of the set of vectors $x_{0}, x_{1}, x_{2}, \ldots, x_{p_{11}}$ and it must also be a linear combination of the set of vectors $x_{1}^{*}$, $\mathrm{x}_{2}^{*}, \ldots, \mathrm{x}_{\mathrm{p}_{21}}^{*} \cdot \mathrm{This}^{*}$ means that:

$$
\left\{\begin{aligned}
x & =\alpha_{0} x_{0}+\alpha_{1} x_{1}+\alpha_{2} x_{2}+\ldots+\alpha_{p_{11}}-x_{p_{11}}-1 \\
& =\lambda_{1} x_{1}^{*}+\lambda_{2} x_{2}^{*}+\lambda_{3} x_{3}^{*}+\ldots+\lambda_{p_{21}}-x^{*} p_{21}^{* 1}
\end{aligned}\right.
$$

Utilizing ( 3.5$)$ we see that (3.7) implies:

$$
\left\{\begin{array}{l}
\left.\alpha_{0}\left(v_{0}+w_{0}\right)+\alpha_{1}\left(v_{1}+w_{1}\right)+\alpha_{2}\left(v_{2}+w_{2}\right)+\ldots+\alpha_{p_{11}}-{ }^{\left(v_{p_{1 I}}-1\right.}{ }^{+w_{p_{1 I}}}\right) \\
=\lambda_{1}\left(y_{1}+z_{1}\right)+\lambda_{2}\left(y_{2}+z_{2}\right)+\ldots+\lambda_{p_{21}-1}\left(y_{p_{21}-1}+z_{p_{2 I}}\right)
\end{array}\right.
$$

Irrom the definition of the vectors $v_{0}^{\prime}, w_{0}^{\prime}, v_{i}^{\prime}, w_{i}^{\prime}, y_{j}^{\prime}$ and $z$ it follows that: 
$(3.9)$

$$
\left\{\begin{array}{l}
\alpha_{0} \mathrm{v}_{0}+\alpha_{1} \mathrm{v}_{1}+\alpha_{2} \mathrm{v}_{2}+\ldots+\alpha_{\mathrm{p}_{11}-1} \mathrm{v}_{\mathrm{p}_{11}-1}=\lambda_{1} \mathrm{y}_{1}+\lambda_{2} \mathrm{y}_{2}+\ldots+\lambda_{\mathrm{p}_{21}-1} \mathrm{y}_{\mathrm{p}_{2 I}-1} \\
\alpha_{0} \mathrm{w}_{0}+\alpha_{1} \mathrm{w}_{1}+\alpha_{2} \mathrm{w}_{2}+\ldots+\alpha_{\mathrm{p}_{11}-1 \mathrm{w}_{11}-1}=\lambda_{1} \mathrm{z}_{1}+\lambda_{2} \mathrm{z}_{2}+\ldots+\lambda_{\mathrm{p}_{21}}-\mathrm{z}_{\mathrm{p}_{21}}-1
\end{array}\right.
$$

Hence for dim $\left(x+x^{*}\right)$ to be less than $p_{11}+p_{11}-1$, the two equations in (3.9) must be satisfied non-trivially.

The above development immediately leads us to:

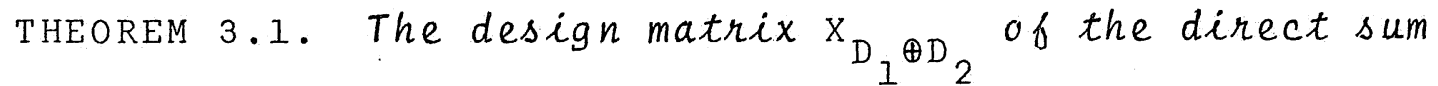
design $\mathrm{D}_{1} \oplus \mathrm{D}_{2}$ is of full column rank if and only if the two equations in (3.9) are not satisfied non-trivially.

The meaning of this theorem is that if the matrices $A_{1}$ and $\mathrm{A}_{2}$ in (3.1) are chosen such that the vectors in (3.4) do not satisfy the equations in (3.9) then the resulting direct sum will be capable of providing unbiased estimates of $\beta_{11} \cup \beta_{2 I}$. A sufficient condition for a nonsingular construction is provided in the following corrollary.

CORROLLARY 3.1. If $D_{1}$ and $D_{2}$ are designs such that $B_{11}$ and $B_{21}$ are estimable in the separate $k_{1}^{m_{1}}$ and $k_{2}{ }_{2}^{2}$ symmetrical factorials respectively, and $A_{1}$ and/or $A_{2}$ are chosen to consist of repetitions of a fixed treatment combination then the direct sum design $\mathrm{D}_{1} \oplus \mathrm{D}_{2}$ is such that $\beta_{11} \cup \beta_{21}$ is estimable in the mixed ${ }^{\mathrm{m}_{1}}{ }^{{ }^{1}}{ }_{\mathrm{k}_{\mathrm{k}}}{ }_{2}^{\mathrm{m}}$ factorial. 
PROOF: Suppose that $A_{1}$ consists of repetitions of a fixed treatment combination from the $k_{1}{ }^{1}$ symmetrical factorial and that $\mathrm{A}_{2}$ consists of arbitrary treatment combinations from the $\mathrm{k}_{2}^{2}$ factorial. Then from (3.2) and (3.3) it follows that each column of $G$ is a constant vector, i.e. a vector whose entries are equal to a scalar. This implies

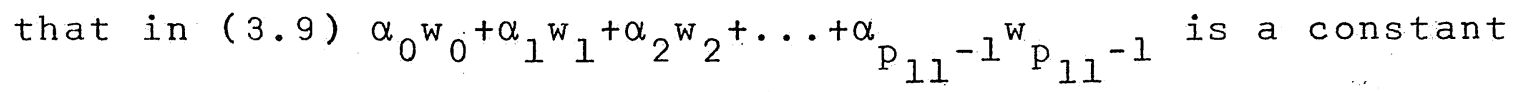
vector, which in turn implies that $z_{1}, z_{2}, \ldots, z_{p_{21}-1}$ are linearly dependent. This contradicts the hypothesis of independence of the $z^{\prime} s$ guaranteed by $\mathrm{D}_{2} \cdot$ Hence the second equation in ( 3.9 ) cannot be satisfied, so that by Theorem

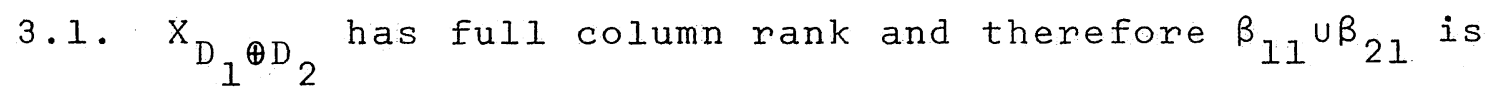
estimable.

A similar argument will show that the first equation in (3.9) cannot be satisfied if $\mathrm{A}_{2}$ consists of repetftions of a fixed treatment combination from the $\mathrm{k}_{2}^{\mathrm{m}}{ }^{2}$ symmetrical factorial. Finally, if both $\mathrm{A}_{1}$ and $\mathrm{A}_{2}$ consist of repetitions of a fixed treatment combination from their respective factorials, then both equations of (3.9) cannot be satisfied and hence $\mathrm{X}_{\mathrm{D}_{1} \oplus \mathrm{D}_{2}}$ will be of full rank. This completes the proof. 
REMARK 3.1. The question arises, whether one may start with $\mathrm{a} \mathrm{D}_{1}$ or $\mathrm{D}_{2}$ in (3.1) such that the design matrices $E$ or $\mathrm{H}$ are less than full column rank and by suitable choices of $A_{1}$ and $A_{2}$ still come up with a direct sum design $D_{1} \oplus D_{2}$ such that $X_{D_{1} \oplus D_{2}}$ is of full. column rank. That this is possible is illustrated in the following example.

$$
\text { Consider the } 2^{2} \text { and } 3^{2} \text { factorials and suppose that we }
$$
are interested in main effects only under the orthogonal polynomial set up. Consider the direct sum design:

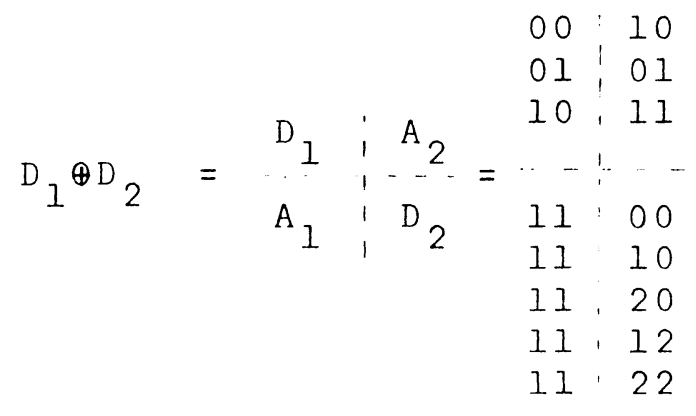

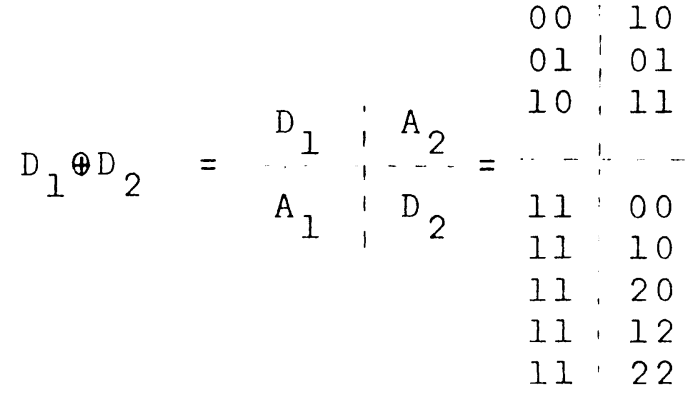

$$
\begin{aligned}
& \text { (3.10) }
\end{aligned}
$$

Note that the design $D_{2}$ gives to rise a design matrix which has rank less than $P_{21}=2(2)+1=5$, since in the $3^{2}$ orthoronal polynomial set up the design matrix 
$(3.11)$

$$
X_{D}=\left[\begin{array}{ccccc}
\frac{1}{3} & -\frac{1}{\sqrt{6}} & \frac{1}{3 \sqrt{2}} & -\frac{1}{\sqrt{6}} & \frac{1}{3 \sqrt{2}} \\
\frac{1}{3} & 0 & -\frac{2}{3 \sqrt{2}} & -\frac{1}{\sqrt{6}} & \frac{1}{3 \sqrt{2}} \\
\frac{1}{3} & \frac{1}{\sqrt{6}} & \frac{1}{3 \sqrt{2}} & -\frac{1}{\sqrt{6}} & \frac{1}{3 \sqrt{2}} \\
\frac{1}{3} & 0 & -\frac{2}{3 \sqrt{2}} & \frac{1}{\sqrt{6}} & \frac{1}{3 \sqrt{2}} \\
\frac{1}{3} & \frac{1}{\sqrt{6}} & \frac{1}{3 \sqrt{2}} & \frac{1}{\sqrt{6}} & \frac{1}{3 \sqrt{2}}
\end{array}\right]
$$

clearly has linearly dependent columns. However, the design matrix $X_{D_{1} \oplus D_{2}}$ of the direct sum design $D_{1} \oplus D_{2}$ is of full column rank, namely $\mathrm{p}_{11}+\mathrm{p}_{21}-1=3+5-1=7$, since

$(3.12)$

$$
\left[\begin{array}{ccccccc}
\frac{1}{6} & -\frac{1}{6} & -\frac{1}{6} & 0 & -\frac{1}{3 \sqrt{2}} & -\frac{1}{2 \sqrt{6}} & \frac{1}{6 \sqrt{2}} \\
\frac{1}{6} & -\frac{1}{6} & \frac{1}{6} & -\frac{1}{2 \sqrt{6}} & \frac{1}{6 \sqrt{2}} & 0 & -\frac{1}{3 \sqrt{2}} \\
\frac{1}{6} & \frac{1}{6} & -\frac{1}{6} & 0 & -\frac{1}{3 \sqrt{2}} & 0 & -\frac{1}{3 \sqrt{2}} \\
\frac{1}{6} & \frac{1}{6} & \frac{1}{6} & -\frac{1}{2 \sqrt{6}} & \frac{1}{6 \sqrt{2}} & -\frac{1}{2 \sqrt{6}} & \frac{1}{6 \sqrt{2}} \\
\frac{1}{6} & \frac{1}{6} & \frac{1}{6} & 0 & -\frac{1}{3 \sqrt{2}} & -\frac{1}{2 \sqrt{6}} & \frac{1}{6 \sqrt{2}} \\
\frac{1}{6} & \frac{1}{6} & \frac{1}{6} & \frac{1}{2 \sqrt{6}} & \frac{1}{6 \sqrt{2}} & -\frac{1}{2 \sqrt{6}} & \frac{1}{6 \sqrt{2}} \\
\frac{1}{6} & \frac{1}{6} & \frac{1}{6} & 0 & -\frac{1}{3 \sqrt{2}} & \frac{1}{2 \sqrt{6}} & \frac{1}{6 \sqrt{2}} \\
\frac{1}{6} & \frac{1}{6} & \frac{1}{6} & \frac{1}{2 \sqrt{6}} & \frac{1}{6 \sqrt{2}} & \frac{1}{2 \sqrt{6}} & \frac{1}{6 \sqrt{2}}
\end{array}\right]
$$

has seven linearly independent columns. 


\section{CLASSES OF SIMILAR DESIGNS}

In this section we show that designs obtained via the sum composition method can be used to produce classes of similar designs if the parametric vector $\beta_{11} \cup \beta_{21}$ satisfies certain regularity conditions, which are typically met in many practical applications.

Let us now apply some of the ideas laid down in two recent papers by Srivastava, Raktoe and Pesotan [1970] and by Pesotan, Raktoe and Federer [1972].

In section 2, we introduced the set $G$ as the set of treatment combinations of order $\prod_{i=l}^{t} k_{i}$. In the present setting $G$ has cardinality $\left(k_{1}{ }^{l}\right)\left(k_{2}{ }^{2}\right)$, since we are dealing with direct sum designs from two symmetrical factorials. We denote an element of $G$ by the $\left(m_{1}+m_{2}\right)$-tuple $\left(x_{11}, x_{12}, \ldots, x_{1 m_{1}}, x_{21}\right.$, $x_{22}, \ldots, x_{2 m_{2}}$ ), where $x_{i j}$ is an element of $G_{i}=\left\{0,1,2, \ldots, k_{i}-1\right\}$, $i=1,2, j=1,2, \ldots, m_{i}$. Further, let an element of $\theta=\beta_{1} \cup \beta_{2}$ be

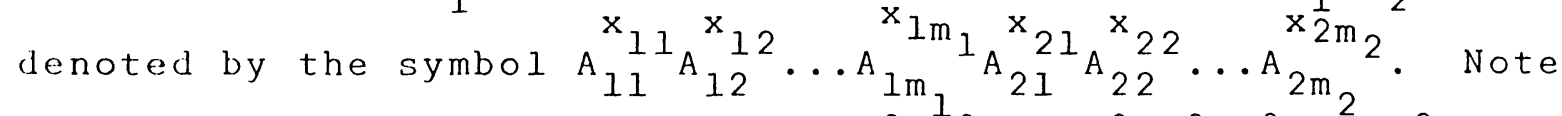
that in this notation the mean $\mu=A_{11}^{0} A_{12}^{1} \ldots A_{m_{1}}^{0} A_{21}^{0} A_{22}^{0} \cdots A_{2 m}^{0}$ und $\left\{\Lambda_{11}^{1} A_{12}^{0} \ldots A_{1 \mathrm{~m}_{1}}^{0} \Lambda_{21}^{0} A_{22}^{0} \ldots A_{2 m_{2}}^{0}, A_{11}^{2} A_{12}^{0} \cdots A_{1 m_{1}}^{0} A_{21}^{0} A_{22}^{0} \cdots A_{2 m_{2}}^{0}\right.$, $\left.\ldots A_{11}^{k_{1}-1} A_{12}^{0} \ldots A_{1 m_{1}}^{0} A_{21}^{0} A_{22}^{0} \ldots A_{2 m_{2}}^{0}\right\}$ represents the set of $k_{1}-1$ normalized orthogonal parametric contrasts associated with the first factor, etc. 


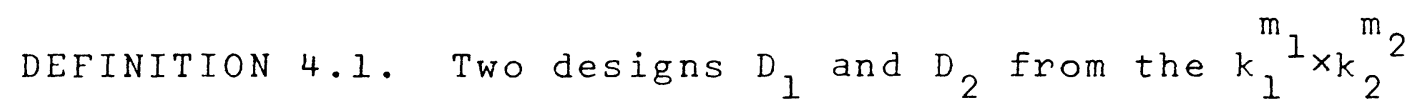
mixed factorial are said to be similar with respect to a subvector of $\theta$ if and only if the underlying infirmation matrices $X_{D_{1}}^{\prime} X_{D_{1}}$ and $X_{D_{2}}^{\prime} X_{D_{2}}$ have the same spectrum.

DEFINITION 4.2. A parametric sub vector $\theta^{*}$ of $\theta$ is said

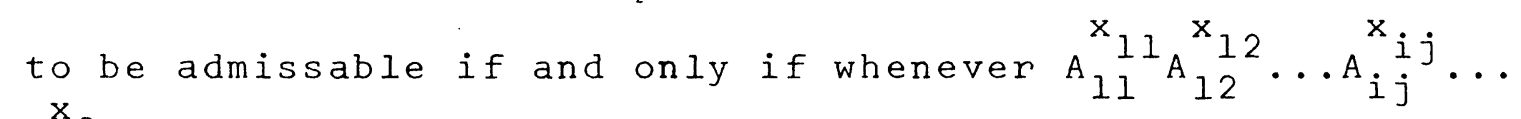
${ }^{A}{ }_{2 m} m_{2}$ belongs to $\theta^{*}$ and $x_{i j} \neq 0$, then $A_{11}^{x_{11} A_{12} x_{12}} \ldots A_{i j}^{r} \ldots A_{2 m_{2}}^{x_{2} m_{2}}$ belongs to $\theta^{*}$ for all $r \neq 0$.

Let $\Omega$ be the group of level permutations acting on the elements of $G$. An element $\omega$ of $\Omega$ is of the form

$(4.1)$

$$
w=\left(w_{1}, w_{2}\right)=\left(w_{11}, w_{12}, \ldots, w_{1 m_{1}}, w_{21}, w_{22}, \ldots, w_{2 m_{2}}\right)
$$

such that: $\omega_{i}=\left(\omega_{i I}, \omega_{i 2}, \ldots, \omega_{i m_{i}}\right)$ and $\omega_{i j}$ is a permutation acting on $G_{i}, i=1,2, j=1,2, \ldots, m_{i}$.

The following lemmas are special cases from srivastava, Raktoe and Pesotan [1971].

LEMMA 4.1. If $\mathrm{D}$ is an arbitrary design from the $\mathrm{k}_{1}^{\mathrm{m}^{1}} \times \mathrm{k}_{2}^{\mathrm{m}} 2$ mixed factorial and $\omega(D)$ is the permuted design obtained from 1 ) and $\theta^{*}$ is admissible, then there exists an orthogonal matrix '' such that

$$
(4.2)
$$

$$
x_{\omega(D)}=x_{D}{ }^{P} \omega
$$

where $x_{1}$ and $x_{\omega}(D)$ are design matrices of the fractions $D$ and $\omega(D)$ with respect to $\theta^{*}$. 
LEMMA 4.2. If $\theta^{*}$ and $\theta^{* *}$ are two admissable subvectors of $\theta$, then the vector $\theta^{*} \cup \theta^{* *}$ obtained by set theroetic union of $\theta^{*}$ and $\theta^{* *}$ is also admissable.

These two lemmas together with the definition of $\omega$ in (4.1) lead us immediately to the following theorem.

THEOREM 4.1. Let $\mathrm{D}_{1}$ and $\mathrm{D}_{2}$ be two fractions from the ${ }_{k}{ }_{1}{ }^{2}$ and $k_{2}^{{ }^{m}}$ factorial respectively for the admissable vectors $\beta_{11}$ and $\beta_{21}$. Let $\omega_{1}\left(D_{1}\right)$ and $\omega_{2}\left(D_{2}\right)$ be the fractions obtained from $\mathrm{D}_{1}$ and $\mathrm{D}_{2}$ be the actions of $\omega_{1}$ and $\omega_{2}$ respectively. If

$$
D_{1} \oplus D_{2}=\begin{array}{l:l}
D_{1} & A_{2} \\
\hdashline A_{1} & D_{2}
\end{array} \text { and } \omega_{1}\left(D_{1}\right) \oplus \omega_{2}\left(D_{2}\right)=\begin{gathered}
\omega_{1}\left(D_{1}\right) \\
\omega_{1}\left(A_{1}\right)
\end{gathered} \omega_{2}\left(\omega_{2}\left(D_{2}\right)\right.
$$

are the two direct sum designs for $B_{11} \cup \beta_{21}$ obtained by sum composition and action of $\omega_{1}$ and $\omega_{2}$ respectively then $\mathrm{D}_{1} \oplus \mathrm{D}_{2}$ and $\omega_{1}\left(D_{1}\right) \oplus \omega_{2}\left(D_{2}\right)$ are similar designs, $i . e$. the underlying information matrices $x_{D_{1} \oplus D_{2}}^{\prime} x_{D_{1} \oplus D_{2}}$ and $x_{\omega_{1}}^{\prime}\left(D_{1}\right) \oplus \omega_{2}\left(D_{2}\right)$ $x_{\omega_{1}}\left(D_{1}\right) \oplus \omega_{2}\left(D_{2}\right)$ have the same characteristic roots.

PROOF: By lemma 4.2. we know that $\beta_{11} \cup \beta_{21}$ is admissable and from (4.1) we know that $\omega=\left(\omega_{1}, \omega_{2}\right)$, which implies that $\omega_{1}\left(D_{1}\right) \oplus \omega_{2}\left(I_{2}\right)=\omega\left(D_{1} \oplus D_{2}\right)$. Hence by lemma 4.1 there exists an orthoronal matrix $\mathrm{P}_{\omega}$ such that 
$(4.4)$

$X_{\omega\left(D_{1} \oplus D_{2}\right)}=X_{D_{1} \oplus D_{2}}{ }^{P} \omega$

Therefore the underlying information matrices of $D_{1} \oplus D_{2}$ and $\omega\left(D_{1} \oplus D_{2}\right)$ have the same spectrum.

The meaning of theorem 4.1 is that when a design is obtained using the sum composition method one has also obtained a class of designs each one providing the same amount of information, if this quantity is taken as a functional on the spectrum of the information matrix. In a recent paper Pesotan, Raktoe and Federer [1972] have obtained some characterization and enumeration results on classes of similar designs in a very general setting. These results can be applied in the present setting as well.

As an illustration of theorem 4.1 consider the direct sum design for main effects of the $2^{2} \times 3^{2}$ factorial discussed in the introductory part of this paper. If $\omega$ is taken to be $\omega=\left(\omega_{1}, \omega_{2}\right)=\left(\omega_{11}, \omega_{12}, \omega_{21}, \omega_{22}\right)$ such that:

$(4.5)$

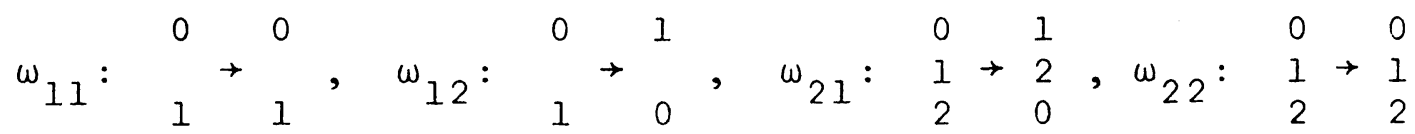

then the designs $D_{1} \oplus D_{2}$ and $\omega_{1}\left(D_{1}\right) \oplus \omega_{2}\left(D_{2}\right)$ below: 


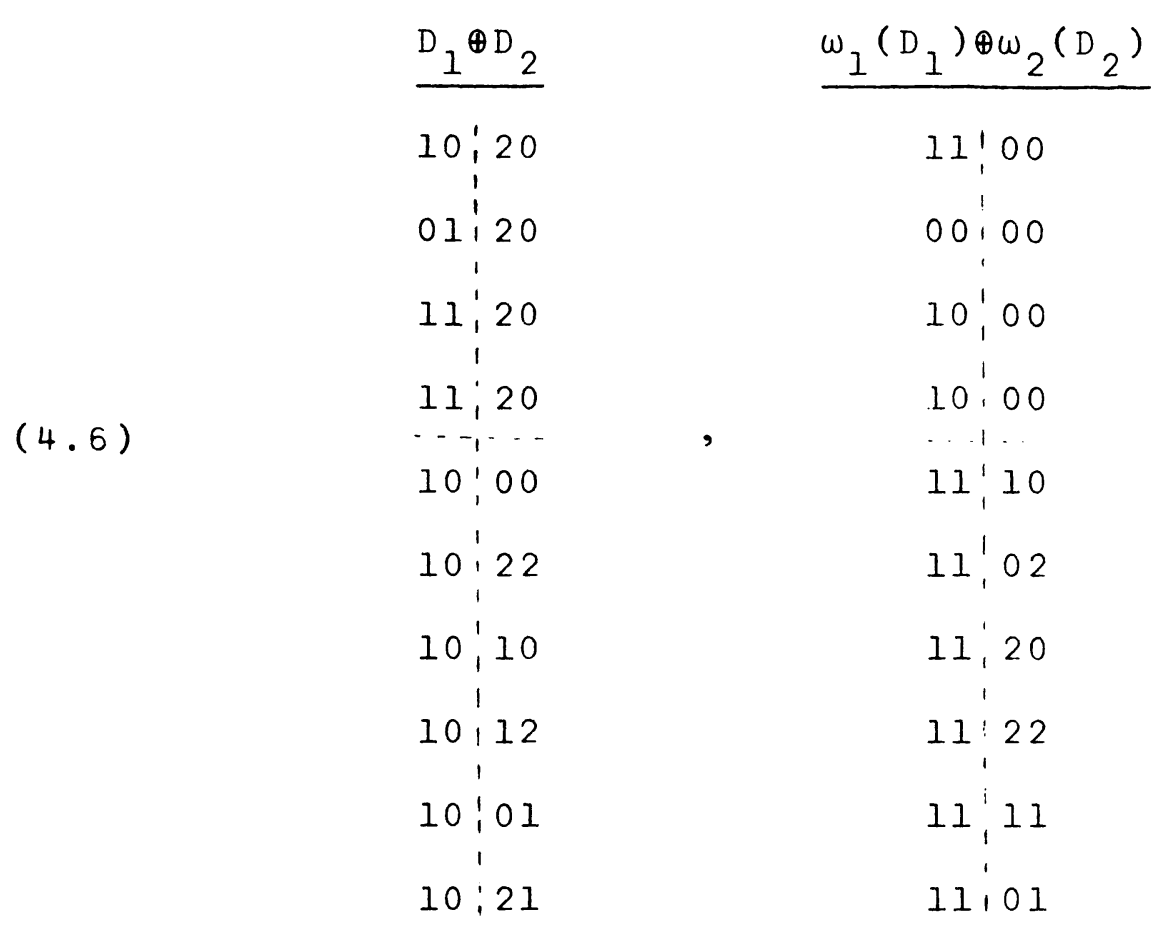

are such that the underlying information matrices have the same spectrum.

\section{A MODIFIED SUM COMPOSITION METHOD}

The sum composition method as discussed in section 3 results always in a direct sum design which has one treatment combination too many. This comes from the fact that in the individual designs each time the mean is to be estimated. In the saturated case the number of treatment combinations should equal the number of parameters to be estimated in the mixed factorial, i.e. $\mathrm{N}_{1}+\mathrm{N}_{2}$ should be equal to $\mathrm{P}_{11}+\mathrm{P}_{21}-1$. The direct sum procedure of section 3 would in this case provide Pl 1 + 12 treatment combinations, i.e. one two many. 
Consider the design $D_{i}$ such that $P_{i l}$ parameters are estimable in the $k_{i}{ }_{i}$ factorial. Suppose that $\beta_{i l}$ is admissable and that $D_{i}$ contains the treatment combination $\left(x_{i 1}^{*}, x_{i 2}^{*}, \ldots, x_{i m_{i}}^{*}\right), i=1,2$. From the development in the previous chapter we know that a similar design $\omega_{i}^{*}\left(D_{i}\right)$ can be obtained from $D_{i}$ such that when the permutation $\omega_{i}^{*}$ is applied to each of the treatment-combinations in $D_{i}$ it takes $\left(x_{i 1}^{*}, x_{i 2}^{*}, \ldots, x_{i m_{i}}^{*}\right)$ into $(0,0, \ldots, 0)$. Hence $\omega_{1}^{*}\left(D_{1}\right)$ and $\omega_{2}^{*}\left(D_{2}\right)$ can be written as:

(5.1) $\frac{\omega_{1}^{*}\left(D_{1}\right)}{00 \ldots 0} \quad \frac{\omega_{2}^{*}\left(D_{2}\right)}{D_{1}^{*}} \quad \frac{00 \ldots 0}{D_{2}^{*}}$

where $D_{i}^{*}$ consists of the remainder of the permuted treatment combinations of $\omega_{i}^{*}\left(D_{i}\right)$. Following Definition 3.1 we may now define the direct sum of designs $D_{1}$ and $D_{2}$ as:

$$
\mathrm{D}_{1} \oplus \mathrm{D}_{2}=\omega_{1}^{*}\left(\mathrm{D}_{1}\right) \oplus \omega_{2}^{*}\left(\mathrm{D}_{2}\right)=\begin{array}{c:c}
\mathrm{D}_{1}^{*} & \mathrm{~A}_{2} \\
\hdashline 00 \ldots 0 & 00 \ldots 0 \\
\hdashline \mathrm{A}_{1} & \mathrm{D}_{2}^{*} .
\end{array}
$$

where $A_{i}$ consists of $N_{i}^{-1}$ treatment combinations from the ${ }_{k_{i}}^{\mathrm{m}}$ factorial. Note, that such a direct sum design has $\mathrm{N}_{1}-1+\mathrm{N}_{2}-1+1=\mathrm{N}_{1}+\mathrm{N}_{2}-1$ treatment combinations, yielding a saving of one treatment combination over the design in (3.1). 
As an illustration, consider the designs introduced in the introduction and further discussed in section 4 . Taking the permutations $\omega_{1}^{*}=\left(\omega_{11}^{*}, \omega_{12}^{*}\right), \omega_{2}^{*}=\left(\omega_{21}^{*}, \omega_{22}^{*}\right)$ as:

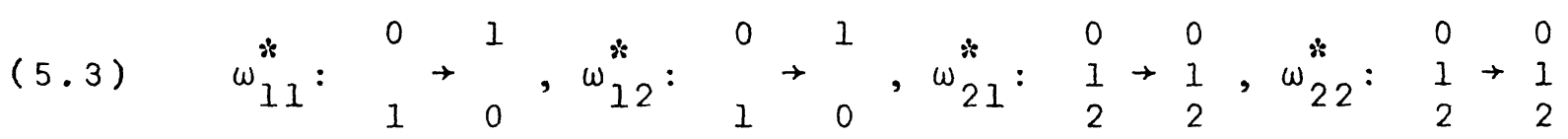

we obtain:

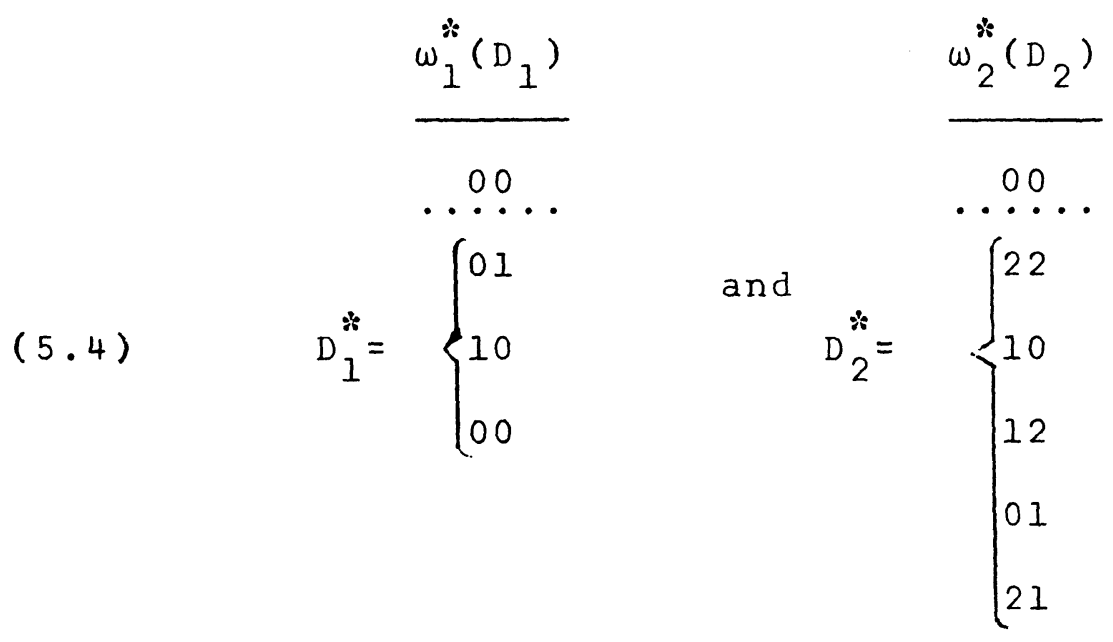

Utilizing (5.2) we then may produce a direct sum design in the following way:

$(5.5)$

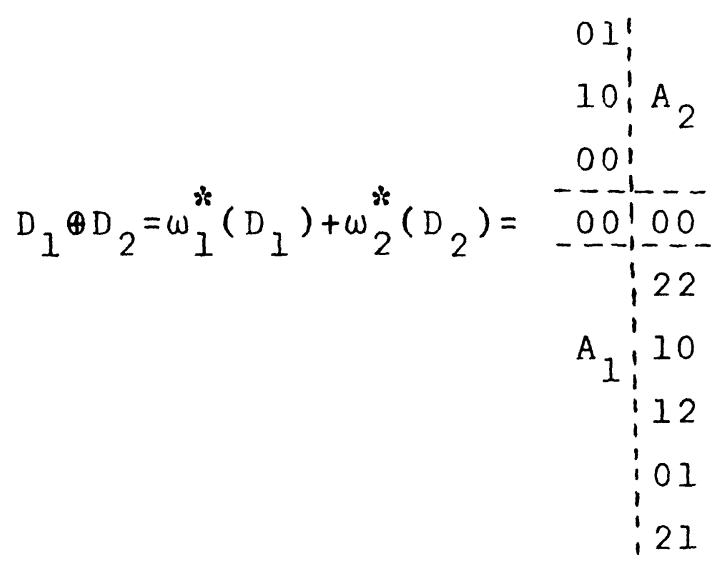


where $A_{1}$ consists of $\mathrm{N}_{1}-1=6-1=5$ treatment combinations from the $2^{2}$ factorial and $A_{2}$ consists of $\mathrm{N}_{2}-1=4-1=3$ treatment combinations from the $3^{2}$ factorial.

As a further example, consider the main effect plans:

(5.6) $\quad D_{1}=\begin{aligned} & 0 \\ & 1 \\ & 2 \\ & 3\end{aligned}, \begin{aligned} & 1000 \\ & 0111 \\ & 1011 \\ & 0001 \\ & 0010\end{aligned}$

from the $4^{1}$ and $2^{4}$ symmetrical factorials respectively. Let

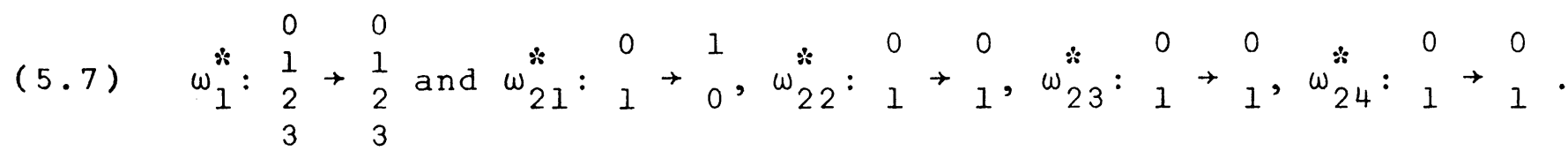

then

$(5.8)$

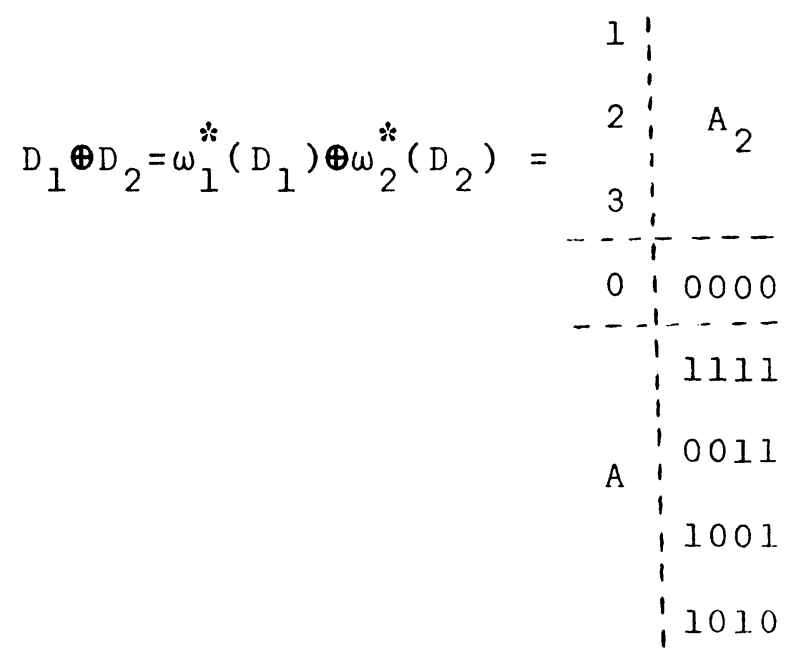

is a direct sum design consisting of 8 treatment combinations from the $4 \times 2^{4}$ mixed factorial if $A_{1}$ and $A_{2}$ are chosen properly. For example, if

$$
A_{1}=\begin{aligned}
& 0 \\
& 1 \\
& 2 \\
& 3
\end{aligned}, \text { and } A_{2}=\begin{aligned}
& 0101 \\
& 0110 \\
& 1100
\end{aligned}
$$


then the resulting direct sum design provides an orthogonal main effect plan in the sense of Addelman [1972], whose design on page 104 suggested the selected $A_{1}$ and $A_{2}$.

The algebraic and construction aspects of the direct sum design in (5.2) can be explored along the same lines as in Theorem 3.1 and Corollary 3.1 .

\section{SOME DESIRABLE PROPERTIES}

Sofar in the development we have ignored the various properties, which come typically into play when dealing with fractional replication. The first one is the property of orthogonality. Suppose that this property is imposed on the direct sum design $D_{1} \oplus D_{2}$ in (3.1). This means that

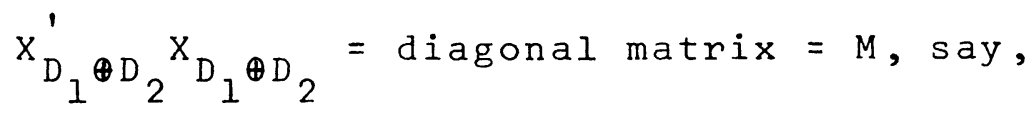

so that from (3.3) we must have:

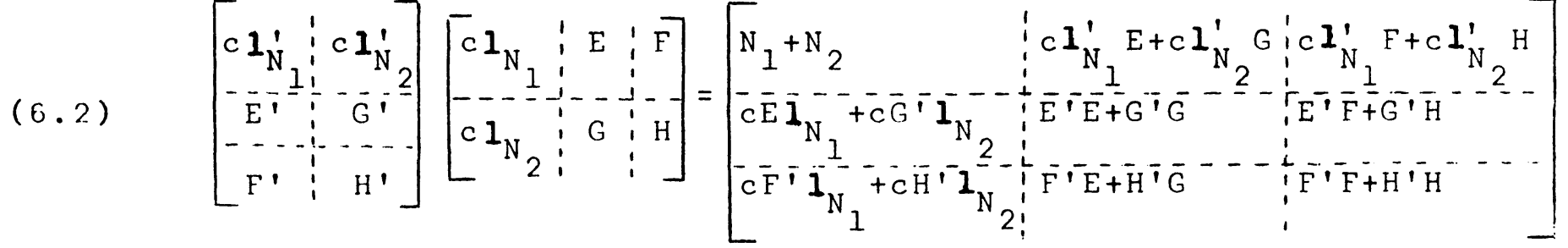

$$
=\left[\begin{array}{c:c:c}
C^{*} & 0 & 0 \\
\hdashline 0 & M & 0 \\
\hdashline 0 & 0 & M_{2}
\end{array}\right]
$$


where $c^{*}=\mathrm{N}_{1}+\mathrm{N}_{2}$ and the $\mathrm{M}_{i}$ 's are diagonal matrices of appropriate dimensions comprising M. Equation (6.2) implies the following conditions:

$(6.3)$

$$
\left\{\begin{array}{l}
E^{\prime} E+G^{\prime} G=M_{1} \\
F^{\prime} F+H^{\prime} H=M_{2} \\
I_{N_{1}}^{\prime} E+I_{N_{2}}^{\prime} G=0 \\
I_{N_{1}} F+I_{N_{2}}^{\prime} H=0 \\
E^{\prime} F+G^{\prime} H=0
\end{array}\right.
$$

By suitable selections of designs $D_{1}, D_{2}, A_{1}$ and $A_{2}$ in (3.1) one may satisfy the conditions in (6.3) and obtain an orthogonal direct sum design. It is clear that the conditions in ( 6.3 ) are in addition to the condition that the equations in (3.9) are not satisfied.

Along these same lines conditions can be formulated such that the direct sum design $D_{1} \oplus D_{2}$ is variance balanced or bias balanced [see Hedayat, Raktoe and Federer [1972]). These properties along with the orthogonality property becomes especially meaningful, if we are dealing with special designs, such as the resolution III and $V$ designs. 


\section{DISCUSSION}

As is apparent from section 6 we just have initiated thoughts on the direct sum method. Considerable work is necessary to exploit in full the ramifications of this method, especially in the odd and even resolution settings.

\section{LITERATURE CITED}

1. Addelman, S. (1972). Recent developments in the design of factorial experiments. J. Amer. Statist. Assoc. 67, $103-110$.

2. Chakravarti, I.M. (1956). Fractional replication in asymmetrical factorial designs and partially balanced arrays. Sankhya, $17,143-164$.

3. Hedayat, A., Raktoe, B. L. and Federer, W. T. (1972) On a measure of bias due to fitting an incomplete model. Submitted for publication in the Annals of Statistics.

4. Pesotan, H., Raktoe, B. L. and Federer, W. T. (1972). On complexes of Abelian groups with applications to fractional factorial designs. Submitted for publication in the Annals of Statistics.

5. Srivastava, J.N., Raktoe, B.L. and Pesotan, H. (1971). On invariance and randomization in fractional replication In the process of publication with the Annals of Statistics.

6. Rao, C. R. (1947). Factorial experiments derivable from combinatorial arrangements in arrays. J. Roy. Stat. Soc. Suppl.) 9, 128-139. 